membranes at the base of the brain. Whether this was of the nature of a gummatous inflammation is an open question. The cerebral symptoms certainly diminished in intensity before constitutional treatment was resumed, which fact speaks against specific origin. On the other hand, it is contrary to usual experience to find a syphilitic inflammation originating at this late period of the disease immediately after some six weeks or more of thorough specific treatment. The neuro-retinitis improved steadily after the specific treatment was resumed, but not as promptly as I would have expected of a purely syphilitic inflammation.

The conclusion seems to me not unreasonable, that the optic neuritis was a sympathetic affection starting from the inflammation in the left eye, and the primary disease would in that light appear to me as a complication of a gummatous deposit in the iris with secondary irido-choriditis of non-specific nature. (To be concluded.)

\section{LACERATION OF THE CERVIX UTERI.}

\section{BY J. HENRY CARSTENS, M.D.,}

PROFESSOR OF OBSTETRICS AND CLINICAL GYNECOLOGY, DETROIT COLLEGE OF MEDICINE.

When the granite monument which may be erected over the future grave of T. A. Emmet, or the marble urn which may hold his ashes, if his body should be cremated, have long yielded to the slow but certain action of the atmosphere and disappeared from view, then the name of Emmet will still be known, and reverently mentioned in connection with laceration of the cervix. Until the last man and woman have disappeared there will be children born, and as long as children are born, tears of the external os will occur, as neither the art and science of the obstetrician nor the vis medicatrix natura can prevent them in all cases. Laceration occurs in cases left entirely to-nature, as well as in the hands of the most careful and experienced accoucheur. We must start with this idea clear in our minds, that the occurrence of laceration is no proof of poor midwifery or meddlesome interference, although manual efforts to hasten. dilation, or the use of the forceps before complete opening of the external os, are a fruitful cause of tears. It is claimed that abortions are also etiological factors. It seems to be the opinion of the majority of the profession that immediate repair is of little avail, as union seldom takes place, and is only indiicated to stop the hæmorrhage when large vessels have been torn.

The symptoms of laceration of the cervix are usually those of some uterine disease: backache, headache, leucorrhoeal discharge, bearing-down pains, etc. The symptoms vary to some degree, whether the laceration has cicatrized or not; in the latter more or less purulent discharge takes place; in the former this is not the case, and often misleads the patient and physicians in thinking there is no uterine disease.

The raw granulating surface has been called an uicer, erosion, etc., until Emmet showed that it was a tear, and how it could be repaired. Lacerations are a frequent cause of subinvolution and displacement of the womb, the latter not subsiding until the proper treatment of the former.

The most troublesome cases are those in which no granulating surface exists, but cicatrization has taken place. Very many of these cases do not require any treatment, as no symptoms are produced. Such cases give the easy-going physician a chance to say that cervical lacerations are of frequent occurrence and do not amount to much, as every woman has a tear if she has given birth to a child. Of course this is partly true; some women have lacerations which have cicatrized and produce no symptoms, but who wants to meddle with such cases? No one ever thinks of treating or operating on cases of that kind. But when we find cases of flexions, versions, descent and enlargements, due to a subinvolution caused by a tear, no pessaries or support, no injection or application will cure the case; only an operation can.

The reflex symptoms produced by cervical laceration are many: such as headaches, spinal irritations, palpitation, neuralgia, disturbances of the stomach, liver and kidneys. These do not come on shortly after the tears occur, but some years later. I believe the cicatrix gradually contracts and compresses some nerve filaments. The cicatrix is often not sensitive to the touch. During the menstrual period the symptoms are generally more marked, varying according to the complications which exist. The sympathetic symptoms and reflex disturbances produced by cercical laceration are almost innumerable, and for that reason the real cause of a woman's illness is often overlooked, and she is treated for almost every disease from acholia to zona.

We must also remember that nearly all observers have called attention to the fact that uterine cancer generally commences at a point of laceration of the cervix, and that we have a right to assume that a long-continued ulcerating tear in the cervix may develop into a malignant growth. Therefore, all cases of cervical laceration which have not healed should be repaired, and those cases which have cicatrized, and produce secondary effects and reflex symptoms, should also be operated on. In my experience, many cases of repeated miscarriages are caused by tears, especially if the latter are deep. Many women anxious to have children can be made happy by an operation for laceration of the cervix. We often meet cases in which women have had a severe labor during the first confinement. The child was born dead, laceration occurred, and no other child was carried to full term, labor always occurring at the third to sixth month. This variety of sterility can be cured.

The operation consists in simply paring the edges, and sewing the raw surfaces carefully together. Considerable discussion has lately taken place on the necessity of removing all cicatricial tissue. Some have even performed a second operation when the cicatricial plug has not been thoroughly removed the first time. For the purpose of making a clean cut and getting away the cicatrix at the angle there is no better instrument than Skene's hawkbill scissors, although a knife and scissors can also be used, espe- 
cially the latter. The sewing can be done with various kinds of needles which have been devised for that purpose, and with silver wire, silk, catgut, or silkworm gut. As the uterine tissue is very firm and it is difficult to get a needle to pass through, I have had Mr. Kuhlman, of Detroit, make a needle with a corkscrew curve on a short handle. This enables all cicatricial tissues.

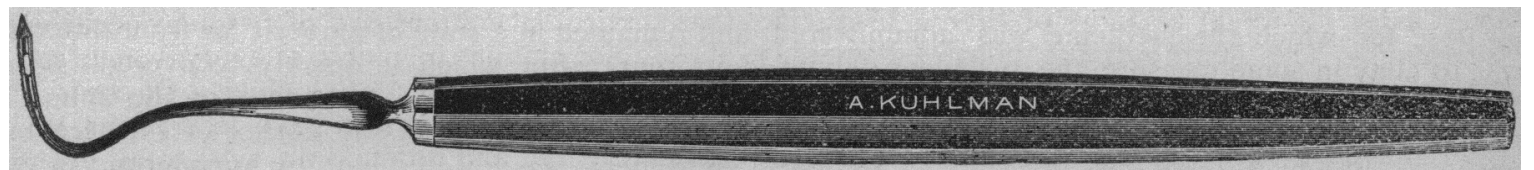

one to put the sutures just where he likes, and fit the raw surfaces most accurately. I have tried the various sutures. Silk prepared as proposed by Skene is very good, but requires removal after union has taken place. Silver wire is good, but difficult to remove unless the patient is again put under an anæsthetic. When silver wire is used, I find it the best plan to put four or five perforaterl shot on the wire, and compress the last one only. This enables one to remove the wire readily by simply cutting it be-

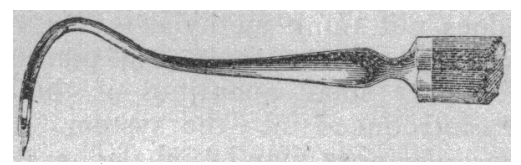

tween the last two shot, pulling all the shot off the wire and grasping the long ends of the latter, simply pulling them out. Catgut is very good, and need not be removed; which is a great advantage. Still, catgut is often unreliable, and is very difficult to tie so that it will hold. I have therefore lately tried silkworm gut, and find it most excellent, if a large size be used and kept thoroughly aseptic. It need not be removed, although it takes a long time to becume absorbed. If the circular artery be cut, it may be necessary in some cases to ligate it, but generally the sutures will stop the hæmorrhage.

The operation for laceration of the cervix is therefore very simple: Cut out a wedge-shaped piece, and remove all cicatricial tissue; carefully adjust the raw surfaces with silkworm gut sutures. I believe in chluroform as an anæsthetic.

After the operation the patient is kept in bed for a week. Use carbolized douches twice a day; but this is not necessary, as I have often paid no attention to the patients except to see that no inflammation developed, and then examined them in five or six days, and if union was perfect, allow them to sit up; in a few days they would follow their ordinary vocation.

The operation is not dangerous unless chronic or subacute peritonitis or cellulitis exist, when no operation should be attempted until after all symptoms have disappeared; and even then great care is needed, as the uterus must be very carefully handled, and not much traction made. In these cases, also, the aftertreatment must be more strict; the patient must be kept quiet and on a light diet; in ordinary cases I allow a full diet.

My conclusions are:

I. All lacerations which have not cicatrized should
3. The wedge-shaped piece can be removed with a knife or scissors, the latter to be preferred.

4. Silkworm gut or catgut is to be preferred, although silk or silver wire may be used.

21 Macomb St., Detroit, May I I, I887.

\section{MEDICAL PROGRESS.}

Strophanthus in Heart-Disease. - Dr. J. Hutchinson, Physician to Anderson's College Dispensary, Glasgow, says: I have administered the drug in twelve cases of heart disease; nine were functional and three organic, and I have much reason to be pleased with the success of the treatment, and with the amount of relief I gave my patients.

On looking over my notes I find two cases of mitral disease, in one of which there was a loud murmur, both obstructive and regurgitant. The patient was a woman, aged 45 , in whom the prominent symptoms were harsh, hacking cough occurring in paroxysms, dyspnœa, and even at times orthopnœa, palpitation, and œedema of feet and legs. The pulse was intermittent, with a regular irregularity, and beating 90 to the minute. Strophanthus was given in half drop doses at first, and was gradually increased until she was taking 2 minims three times a day. Almost from the first dose taken an alteration in the sufferings of the patient was observed. The heart-sounds were firmer and steadier; the pulsebeats, though still irregular, were not so fast; cough was much less troublesome, and the palpitation was neither so frequent nor so violent. Along with this there was a copious increase in the renal secretion, which soon relieved both the visceral engorgement and the odema in the feet and legs. In fourteen days she felt so well as to be able to return to her household duties, In the other case of mitral disease the symptoms were much the same, but not nearly so severe. The same dose was given, and the effect was as satisfactory and rapid.

Another case of aortic stenosis in an old lady, aged 60 , who had for years been a martyr to chronic cough, palpitation, and the other symptoms attendant upon stenosis of the aortic orifice, received great relief from a one-minim dose of the drug. In this case palpitation was very violent, the pulse was rapid, and there was extensive passive congestion of both lungs. EEdema of the feet and legs was also present in a marked degree. Under the influence 